Real Analysis Exchange

Vol. 23(2), 1997-1998, pp. 753-755

Kenneth S. Miller, 25 Bonwit Road, Rye Brook, New York, 10573, USA

Stefan G. Samko, Department of Mathematics and Mechanics, Rostov State University, Rostov-na-Donu, 344104, Russia.

Currently: Universidade do Algarve, UCEH, Campus de Gambelas, Faro,

8000, Portugal, e-mail: ssamko@ualg.pt

\title{
A NOTE ON THE COMPLETE MONOTONICITY OF THE GENERALIZED MITTAG-LEFFLER FUNCTION
}

The Mittag-Leffler function

$$
E_{\alpha}(t)=\sum_{k=0}^{\infty} \frac{t^{k}}{\Gamma(\alpha k+1)}
$$

was introduced early in this century and was studied by various mathematicians. In particular it arises in the study of the fractional calculus [5]. Other applications are discussed in [2], [3]. In particular Pollard [4] proved that the Mittag-Leffler function $E_{\alpha}(-t), t \geq 0$ was completely monotonic, that is

$$
(-1)^{m} \frac{d^{m}}{d t^{m}} E_{\alpha}(-t) \geq 0
$$

for all $m, m=0,1,2, \ldots$ if $0 \leq \alpha \leq 1$.

The generalized Mittag-Leffler function $E_{\alpha, \beta}(x)$ may be defined as

$$
E_{\alpha, \beta}(x)=\sum_{k=0}^{\infty} \frac{x^{k}}{\Gamma(\alpha k+\beta)}, \quad \alpha \geq 0, \quad \beta \geq 0 .
$$

In a recent paper [6] Schneider proved that the generalized Mittag-Leffler function $E_{\alpha, \beta}(-x), x \geq 0$, was completely monotonic for $\alpha>0, \beta>0$ if and only if $0<\alpha \leq 1$ and $\beta \geq \alpha$. His proof is based on the usage of the corresponding probability measures and the Hankel contour integration.

In this note we wish to show that, independent of the fact that in applications it may be of importance to know the probability measure, corresponding

Key Words: Mittag-Leffler function, complete monotonicity

Mathematical Reviews subject classification: 33E20

Received by the editors April 7, 1997 
to the function $E_{\alpha, \beta}(-x)$, the fact itself of complete monotonicity of $E_{\alpha, \beta}(-X)$ for $0 \leq \alpha \leq 1, \beta \geq \alpha$ is an immediate corollary of Pollard's result. This corollary may be obtained purely within the framework of real analysis. The result follows immediately from the following two lemmas.

Lemma 1. $E_{\alpha, \alpha}(-x)=-\alpha \frac{d}{d x} E_{\alpha}(-x), \alpha \geq 0$.

The proof is direct.

Lemma 2. Let $\beta>\alpha>0$. Then

$$
E_{\alpha, \beta}(-x)=\frac{1}{\alpha \Gamma(\beta-\alpha)} \int_{0}^{1}\left(1-t^{\frac{1}{\alpha}}\right)^{\beta-\alpha-1} E_{\alpha, \alpha}(-t x) d t .
$$

Proof. Substitute $E_{\alpha, \alpha}(-t x)$ from (1) into the right-hand side of (2) and interchange the order of integration and summation, which is easy to justify. We obtain

$$
\frac{1}{\alpha \Gamma(\beta-\alpha)} \sum_{k=0}^{\infty} \frac{(-x)^{k}}{\Gamma(\alpha k+\alpha)} \int_{0}^{1} t^{k}\left(1-t^{\frac{1}{\alpha}}\right)^{\beta-\alpha-1} d t
$$

Hence the left-hand side of (2) is easily obtained.

Observe that

$$
E_{0, \beta}(-x)=\frac{1}{\Gamma(\beta)} \cdot \frac{1}{1+x}, \quad \beta>0,
$$

and

$$
E_{0, \beta}(-x)=0, \quad \beta=0 .
$$

In both cases $E_{0, \beta}(-x)$ is completely monotonic.

We note that the formula (2) is a special case of a more general relation due to Dzherbashian [1, p. 120] which states that $x^{\beta+\gamma-1} E_{\alpha, \beta+\gamma}\left(-x^{\alpha}\right)$ is the fractional integral of order $\gamma$ of $x^{\beta-1} E_{\alpha, \beta}\left(-x^{\alpha}\right)$. But we gave the proof of (2) to show that it is very easy.

Now, in view of Lemmas 1 and 2, the complete monotonicity of $E_{\alpha, \beta}(-x)$ with $0 \leq \alpha \leq 1$ and $\beta \geq \alpha$ indeed follows immediately from Pollard's result.

\section{References}

[1] M. M. Dzherbashian, Integral transforms and representations of functions in the complex plane, Nauka, Moscow, 1966 (in Russian).

[2] A. Erdélyi, Higher transcendental functions, vol. III, Robert E. Krieger Publishing Co., 1981. 
[3] K. S. Miller, The Mittag-Leffler and related functions, Integral Transforms and Special Functions 1 (1993), 41-49.

[4] H. Pollard, The completely monotonic character of the Mittag-Leffler function $E_{\alpha}(-x)$, Bull. Amer. Math. Soc. 54 (1948), 1115-1116.

[5] S. Samko, A. Kilbas, and O. Marichev, Fractional integrals and derivatives, Gordon and Breach Science Publishers, 1993.

[6] W. R. Schneider, Completely monotone generalized Mittag-Leffler functions, Expo. Math. 14 (1996), 3-16. 